\title{
Chemotaxis in the human gastric pathogen Helicobacter pylori: different roles for CheW and the three CheV paralogues, and evidence for CheV2 phosphorylation
}

\author{
Marc S. Pittman, Matthew Goodwin and David J. Kelly
}

Department of Molecular Biology and

Biotechnology, University of Sheffield, Firth Court, Western Bank, Sheffield S10 2TN, UK
Author for correspondence: David J. Kelly. Tel: +44114222 4414. Fax: +44 1142728697. e-mail:d.kelly@sheffield.ac.uk

The complete genome sequence of Helicobacter pylori has revealed the presence of a novel set of chemotaxis genes including three cheV paralogues. $\mathrm{CheV}$ is a bi-functional protein, the $\mathbf{N}$-terminal domain being homologous to the signalling-complex linker protein $\mathrm{CheW}$, while the C-terminal domain is homologous to the response-regulator CheY, but its precise function in chemotaxis is unknown. In this study, each of the three cheV paralogues were insertionally inactivated in strain 26695 to determine their importance in the chemotactic signal-transduction pathway of H. pylori. Mutation of HP0019 (cheV1) had a severe inhibitory effect on chemotaxis, as determined by a swarm-plate assay. In contrast, strains carrying single mutations in either cheV2 (HP0616) or cheV3 (HP0393) displayed wild-type swarming behaviour, as did a cheV2/cheV3 double mutant. However, expression of the cheV2 or cheV3 genes in Escherichia coli resulted in an inhibition of chemotaxis in a wild-type strain, indicating their role in chemotaxis, although these genes were unable to complement isogenic $E$. coli cheW or cheY mutants. The product of cheV2/HP0616 was overexpressed in E. coli and purified to homogeneity. Protein fluorescence quenching experiments showed that CheV2 was capable of binding acetyl phosphate, a small-molecule phosphodonor. The measured $\boldsymbol{K}_{\mathrm{m}}$ for acetyl phosphate was $21 \mathrm{mM}$. It is concluded that in the absence of a cheZ gene, the CheV proteins could act as phosphate sinks to control the cellular level of phospho-CheY in $\mathrm{H}$. pylori. However, only CheV1 was critical for chemotaxis, indicating a specific role distinct from the other paralogues in the signal-transduction pathway. Significantly, none of the CheV proteins could substitute for the loss of CheW, as an $H$. pylori cheW null mutant was nonchemotactic.

Keywords: cheY, cheW, cheV, motility, fluorescence, acetyl phosphate, phosphorylation

\section{INTRODUCTION}

The human gastric pathogen Helicobacter pylori is the aetiological agent of chronic active type B gastritis (Marshall et al., 1985) and is strongly associated with gastric and duodenal ulceration (Moss \& Calam, 1992; Wyatt et al., 1987). Long-term infection with $H$. pylori is also a risk factor for gastric cancer (Forman et al., 1991). The importance of motility as a virulence factor in this organism has been firmly established, and several studies have shown that aflagellate, non-motile mutants are unable to colonize animal models (Eaton $e t$ al., 1992, 1996). More recently, it has been established that chemotaxis, the behavioural process which allows the cell to move towards and away from attractants and repellents respectively, is also an essential virulence factor (Foynes et al., 2000).

The paradigm of the bacterial chemotactic response is the system found in enteric bacteria such as Escherichia coli and Salmonella typhimurium, and has been extensively reviewed (Bourret et al., 1991; Blair, 1995; Stock \& Surette, 1996). It has become clear, however, that many bacteria have more complex chemotaxis 
systems than the enterics, with additional or duplicated genes (Armitage \& Schmitt, 1997). The complete genome sequencing of two strains of $H$. pylori (Tomb et al., 1997; Alm et al., 1999) revealed the presence of six che gene homologues. These are: the response regulator gene cheY (HP1067/JHP358), a histidine kinase gene cheA (HP0392/JHP989), cheW, encoding the ternarycomplex-forming protein CheW (HP0391/JHP990) and three unlinked copies of $c h e V$. The latter form a paralagous family and have been designated cheV1 (HP0019/JHP17), cheV2 (HP0616/JHP559) and cheV3 (HP0393/JHP988) by Doig et al. (1999). Each cheV gene encodes a protein consisting of an N-terminal domain homologous to $\mathrm{CheW}$ and a C-terminal domain homologous to CheY. At least three genes are present that encode transducer-like receptor proteins, which possess several conserved glutamate residues that could be methylated as part of a stimulus-adaptation system. However, there is no evidence of potential methyltransferase $(c h e R)$ or methylesterase $(c h e B)$ genes, and so the details of adaptation are unclear. The cheA, cheW and cheV 3 genes are arranged in an operon while cheY and the two further copies of cheV are located at unlinked sites on the chromosome. Beier et al. (1997) characterized the response regulator gene che $Y$ and showed that it was part of a larger operon containing chemotaxis-unrelated stress-induced genes. The deduced histidine kinase CheA is actually a bi-functional protein in $\mathrm{H}$. pylori, as it is fused to an $\mathrm{N}$-terminal CheY domain (Jackson et al., 1995; Kelly, 1998) and has been redesignated CheAY, encoded by the cheAY gene (Foynes et al., 2000). Thus, H. pylori contains five CheY-like domains on separate proteins, but there is no evidence for a cheZ gene (encoding a protein which enhances the intrinsic CheY phosphatase activity) in this bacterium. Mutants in either cheY or cheAY have been shown to be non-chemotactic (Beier et al., 1997; Foynes et al., 2000).

One of the unusual features of the H. pylori chemotaxis machinery is the presence of the three paralogues of $\mathrm{CheV}$. CheV was first identified as a novel chemotaxis protein in Bacillus subtilis (Frederick \& Helmann, 1994) and partial redundancy with $\mathrm{CheW}$ was reported (Rosario et al., 1994). However, the actual function of $\mathrm{CheV}$ in chemotaxis has not been elucidated. To begin to address this problem in $H$. pylori, we have determined, by insertional mutagenesis, the essentiality for chemotaxis of each of the three cheV paralogues in this bacterium. These genes have also been expressed in $E$. coli wild-type and isogenic $\Delta c h e Y$ and $\Delta c h e W$ mutants to investigate the interaction of the $H$. pylori proteins with the enteric chemotaxis system. In E. coli CheY, aspartate residue D57 is the site of phosphorylation via CheA or small-molecule phosphodonors such as acetyl phosphate (Lukat et al., 1992; McCleary \& Stock, 1994; Da Re et al., 1999). In H. pylori, the CheY domains of each of the $\mathrm{CheV}$ paralogues contain this conserved aspartate, raising the possibility that in the absence of a CheZ homologue, these domains could act as 'phosphate sinks' to control the level of phospho-
CheY. In support of this, we have now shown by fluorescence studies that at least one of the H. pylori $\mathrm{CheV}$ proteins (CheV2) is capable of binding acetyl phosphate.

\section{METHODS}

Bacterial strains, plasmids and growth conditions. The bacterial strains and plasmids used in this study are shown in Table 1. H. pylori was routinely grown in brain heart infusion (BHI) medium (Oxoid) with 1.5\% (w/v) Bacto agar (Difco), supplemented with $7 \%(\mathrm{v} / \mathrm{v})$ lysed horse blood. A selective antibiotic supplement of vancomycin, polymyxin B and amphotericin B, each at a final concentration of $10 \mu \mathrm{g} \mathrm{ml}^{-1}$, was added to all H. pylori growth media. H. pylori was cultured at $37^{\circ} \mathrm{C}$ for $48 \mathrm{~h}$ in a Variable Atmosphere Incubator (VAIN; Don Whitley Ltd) in an atmosphere of $5 \%(\mathrm{v} / \mathrm{v}) \mathrm{CO}_{2}$, $87 \%(\mathrm{v} / \mathrm{v}) \mathrm{N}_{2}$ and $8 \%(\mathrm{v} / \mathrm{v}) \mathrm{O}_{2}$. The medium for swarm analysis of $H$. pylori was BHI base with $0.32 \%$ (w/v) Bacto agar (Difco), supplemented with $7 \%(\mathrm{v} / \mathrm{v})$ sterile calf serum. Excess moisture was carefully dried from the agar surface before use. The plates were incubated at $37^{\circ} \mathrm{C}$ for up to $5 \mathrm{~d}$ in the VAIN cabinet.

E. coli strains for plasmid subcloning and overexpression were grown on Luria-Bertani (LB) agar or in LB broth. Supplements of $100 \mu \mathrm{g}$ ampicillin $\mathrm{ml}^{-1}, 30 \mu \mathrm{g}$ chloramphenicol $\mathrm{ml}^{-1}$ and $30 \mu \mathrm{g}$ kanamycin $\mathrm{ml}^{-1}$ were used as appropriate for the selection and maintenance of plasmids. E. coli swarm assays were carried out over a $6-8 \mathrm{~h}$ period at $30^{\circ} \mathrm{C}$ on LB plates with $0.3 \%(\mathrm{w} / \mathrm{v}$ ) Bacto agar (Difco), using inocula from stationaryphase LB broth cultures. Graded expression of pTM30 constructs was achieved by incorporation of IPTG in $\log$ increments over the range $10^{0}-10^{3} \mu \mathrm{M}$ in the swarm agar plates.

Electron microscopy. Cell suspensions were deposited onto Formvar-coated grids, blotted off and negatively stained by the addition of $1 \%(\mathrm{w} / \mathrm{v})$ phosphotungstic acid to the grids for $3 \mathrm{~min}$. After blotting and air-drying, the grids were viewed and photographed using a Philips CM10 transmission electron microscope.

Preparation and sequencing of DNA. Plasmid DNA for screening clones, sequencing and gene disruption experiments was routinely isolated using anion-exchange resin spincolumns (QIAGEN), according to the manufacturer's instructions. Double-stranded sequencing of plasmid DNA was carried out using Applied Biosystems Taq DyeDeoxy terminator cycle sequencing reactions analysed on an ABI model 373A automated DNA sequencer. Synthesis of custom primers was carried out on an Applied Biosystems model 392 DNA synthesizer. H. pylori total chromosomal DNA was extracted using a modified SDS lysis procedure (Marmur, 1961). Restriction endonucleases, T4 DNA ligase and Taq polymerases were purchased from Promega, and $P f u$ polymerase from Stratagene, and were used according to the manufacturer's instructions.

Construction and characterization of $\boldsymbol{H}$. pylori isogenic insertion mutants. A set of PCR primers was designed to amplify the entire coding regions of the cheW, cheV1, cheV2 and cheV 3 genes, both for insertional activation after cloning of the products into pGEM-3Zf $(-)$ (Promega) and for expression after subcloning into pTM30 (Morrison \& Parkinson, 1994). The primers used were: CheW-F (5'-TCGA $\boldsymbol{G} \boldsymbol{A} \boldsymbol{A} \boldsymbol{T} \boldsymbol{C}$ AAGCAACCAATTAAAAGAT-3'), 
Table 1. Strains and plasmids used in this study

\begin{tabular}{|c|c|c|}
\hline $\begin{array}{l}\text { Strain or } \\
\text { plasmid }\end{array}$ & Genotype or characteristics & Source or reference \\
\hline \multicolumn{3}{|l|}{ E. coli } \\
\hline DH5 $\alpha$ & $\begin{array}{l}\phi 80 \mathrm{~d} l a c Z \Delta \mathrm{M} 15 \Delta(\operatorname{lac} Z \mathrm{Y} A-\arg F) \mathrm{U} 169 \text { supE4 hsdR17 recA1 endA1 gyrA96 thi-1 } \\
\text { relA deoR }\end{array}$ & Stratagene \\
\hline BL21 ( $\lambda \mathrm{DE} 3)$ & 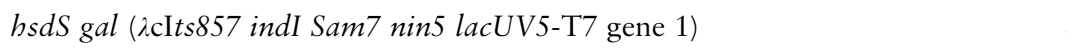 & Studier \& Moffat (1986) \\
\hline RP437 & $\begin{array}{l}\text { thr }(A m)-1 \text { leuB6 his-4 metF }(A m) 159 \text { eda-50 rpsL136 [thi-1 ara-14 mtl-1 xyl-5 } \\
\text { tonA31 tsx-78] }\end{array}$ & P. Hamblin, University of Oxford \\
\hline RP1616 & $\begin{array}{l}\text { (cheZ)DEm67-25 thr (Am)-1 leuB6 his-4 metF(Am)159 eda-50 rpsL136 [thi-1 } \\
\text { ara-14 mtl-1 xyl-5 tonA31 tsx-78] }\end{array}$ & P. Hamblin, University of Oxford \\
\hline RP4606 & $\begin{array}{l}\text { cheW }(\text { Am)113 thr }(A m)-1 \text { leuB6 his-4 rpsL136 [thi-1 (gal-att-L)DE99 ara-14 } \\
\text { lacY1 mtl-1 xyl-5 tonA31 tsx-78] }\end{array}$ & P. Hamblin, University of Oxford \\
\hline RP5232 & $\begin{array}{l}\text { (cheY)DEm60-21 thr }(A m)-1 \text { leuB6 his-4 metF }(A m) 159 \text { rpsL136 [thi-1 ara-14 } \\
\text { lacY1 mtl-1 xyl-5 tonA31 tsx-78] }\end{array}$ & P. Hamblin, University of Oxford \\
\hline \multicolumn{3}{|c|}{ ( } \\
\hline 26695 & Wild-type & Akopyants et al. (1995) \\
\hline MSP100 & 26695 cheV1::cat & This study \\
\hline MSP200 & 26695 cheV2::cat & This study \\
\hline MSP300 & 26695 cheV3::cat & This study \\
\hline MSP400 & 26695 cheV2::aph/cheV3::cat & This study \\
\hline MSP500 & 26695 cheW::cat & This study \\
\hline \multicolumn{3}{|l|}{ Plasmids } \\
\hline рTM30 & Expression vector & Morrison \& Parkinson (1994) \\
\hline pGEM-3Zf $(-)$ & Cloning vector & Promega Ltd \\
\hline pUCAT & pUC18 containing cat cassette from C. coli & Wang \& Taylor (1990) \\
\hline pUKAN & pUC18 containing aphAIII from C. coli & Wang \& Taylor (1990) \\
\hline pMSP1 & pGEM-3Zf $(-)$ containing $H$. pylori cheW inserted into unique HincII site & This study \\
\hline pMSP4 & pGEM-3Zf $(-)$ containing $H$. pylori cheV1 inserted into unique HincII site & This study \\
\hline pMSP5 & pGEM-3Zf $(-)$ containing $H$. pylori cheV3 inserted into unique HincII site & This study \\
\hline pMSP6 & pGEM-3Zf $(-)$ containing $H$. pylori cheV2 inserted into unique HincII site & This study \\
\hline pMSP8 & pTM30 with cheV1 inserted at the BamHI and KpnI sites & This study \\
\hline pMSP9 & pTM30 containing cheW inserted at the $B a m \mathrm{HI}$ and $K p n \mathrm{I}$ sites & This study \\
\hline pMSP10 & pTM30 containing cheV3 inserted at the BamHI and KpnI sites & This study \\
\hline pMSP11 & pTM30 containing cheV2 inserted at the BamHI and KpnI sites & This study \\
\hline pMSP12 & pMSP1 with $c h e W$ inactivated by insertion of cat cassette at unique BsrBR1 site & This study \\
\hline pMSP15 & pMSP4 with cheV1 inactivated by insertion of cat cassette at unique NcoI site & This study \\
\hline pMSP16 & pMSP5 with cheV3 inactivated by insertion of cat cassette at unique BsaBI site & This study \\
\hline pMSP17 & pMSP6 with cheV2 inactivated by insertion of cat cassette at unique $B c l$ I site & This study \\
\hline pMSP18 & $\begin{array}{l}\text { pMSP6 with cheV2 inactivated by insertion of aphAIII cassette at unique } B c l \text { I } \\
\text { site }\end{array}$ & This study \\
\hline
\end{tabular}

CheW-R (5'-TCGAGGT $\boldsymbol{A C C G A A G T C T T T T T T T A A G A - ~}$ TTTC-3'), CheV1-F(5'-TCGAGG $\boldsymbol{A}$ TCCAGCTGATAGTTTAGCGGGC-3'), CheV1-R (5'-TCGAGG $\overline{T A C} C$ TGCTAATTCCAAAAATTG-3'), CheV2-F (5'-TCGAGGATCCAGTAAGAGAGATATTGACAAA-3'), CheV2-R (5'-TCG AGGT $A$ CCTTATGAAAGCGTTTTTTT-3'), CheV3-F (5'-TCGAGG $\boldsymbol{A}$ TCCAGCAGAAAAAACAGCTA-3') and CheV3-R (5'-TCGAG $\overline{G T A C C C G C A T T C T T G T C T A A A A-~}$ T-3'). These primers introduced BamHI or KpnI restriction sites (shown in bold italics) for the in-frame subcloning of these genes into the pTM30 polylinker (see below). The second codon (forward primers) or final codon (reverse primers) of the gene is underlined. PCR reactions were performed with $P f u$ polymerase using genomic DNA from strain 26695 as template.
pGEM-3Zf $(-)$ was linearized with HincII and blunt-end ligated with the products resulting from PCR with the above primers, generating plasmids pMSP1, pMSP4, pMSP5 and pMSP6 (Table 1). These plasmids were linearized at unique restriction sites within the $H$. pylori gene inserts, as detailed in Table 1. Protruding $5^{\prime}$ termini were in-filled using the Klenow fragment of the E. coli DNA polymerase I where necessary. For insertional inactivation, a chloramphenicol acetyltransferase (cat) cassette derived from Campylobacter coli (Wang \& Taylor, 1990) was used. The 900 bp cat cassette excised from pUCAT by HincII digestion was used to inactivate cheW and cheV2. However, it possesses rhoindependent transcription terminators at the $3^{\prime}$ terminus. A non-polar version of this cassette was used to inactivate cheV1 and cheV3. PCR primers Cat-F (5'-TCCGTCGTCGGTAT- 
CGTATGG-3') and Cat-R (5'-TTATCAGTGCGACAAACTGGG-3') were designed to amplify an 829 bp product from pUCAT containing the promoter region of the cat gene but lacking the $3^{\prime}$ transcriptional terminators. Both cassettes were purified using a Gel Extraction Kit (QIAGEN) and blunt-end ligated into the appropriate plasmids. Recombinant clones were selected by growth on LB agar containing $30 \mu \mathrm{g}$ chloramphenicol ml $\mathrm{ml}^{-1}$ and the orientation of the cassettes determined by restriction analysis. The following plasmids were generated: pMSP12, pMSP15, pMSP16 and pMSP17 (see Table 1). To construct a plasmid suitable for generating a $H$. pylori cheV2/cheV3 double mutant, the C. coli-derived aphAIII cassette (Wang \& Taylor 1990) was amplified from pUKAN with $P f u$ polymerase, using primers Kan-F (5'ACTGAGATCTACTCTATGAAGCGCCATATT-3') and Kan-R (5'-CAATAGATCTTTTAGACATCATCTAAATCTAGG-3'). Plasmid pMSP17 was linearized at the unique $B c l$ I site within the cheV2 gene and blunt-end ligated with the aphAIII cassette. E. coli transformants were selected by growth on LB agar containing $30 \mu \mathrm{g}$ kanamycin sulphate $\mathrm{ml}^{-1}$. The resulting plasmid was designated pMSP18.

Transformation of H. pylori 26695 with pMSP12, pMSP15, pMSP16 and pMSP17, and of strain MSP300 with pMSP18, was carried out as previously described (Ferrero et al., 1992). Transformants were selected by addition of $30 \mu \mathrm{g}$ chloramphenicol ml $\mathrm{m}^{-1}$ or $30 \mu \mathrm{g}$ kanamycin sulphate $\mathrm{ml}^{-1}$ to the plating medium as appropriate. Genomic DNA was extracted from putative recombinants, and correct insertion of the resistance cassettes evaluated by PCR using the above primers.

Cloning and expression of $H$. pylori cheW and cheV genes in pTM30 and transformation of $E$. coli chemotaxis mutants. Complementation and expression experiments with the $H$. pylori chemotaxis genes were done by subcloning the cheW, cheV1, cheV2 and cheV3 genes in-frame into the BamHI and $K p n \mathrm{I}$ sites of the expression vector pTM30, and transforming the resulting constructs (Table 1 ) into the E. coli wild-type strain RP437, and chemotaxis mutants RP5232 ( $\Delta$ cheY) and RP4606 $(\Delta c h e W)$. The fidelity of the constructs was checked by DNA sequencing. Expression of proteins was induced with IPTG and monitored by SDS-PAGE on $10 \%$ (w/v) polyacrylamide gels. Swarming and motility phenotypes were determined for the complemented wild-type and mutant strains on LB plus $0 \cdot 3 \%(\mathrm{w} / \mathrm{v})$ Difco agar, containing IPTG in the range $0-1 \mathrm{mM}$.

Purification of the CheV2 protein. pMSP11 was grown in $6 \times 11$ batches of LB plus $100 \mu \mathrm{g}$ ampicillin $\mathrm{ml}^{-1}$ until an $\mathrm{OD}_{600}$ of 0.5 was reached. IPTG was added to $1 \mathrm{mM}$ final concentration and growth continued for $5 \mathrm{~h}$, after which the cells were harvested by centrifugation $\left(8000 \mathrm{~g}, 4^{\circ} \mathrm{C}, 20 \mathrm{~min}\right)$, resuspended in $20 \mathrm{ml} 10 \mathrm{mM}$ HEPES buffer $\mathrm{pH} 7 \cdot 5$ (buffer A) and disrupted in a French press at 12000 p.s.i. $(82 \cdot 8 \mathrm{MPa})$. The broken cells were centrifuged $\left(150000 \mathrm{~g}, 4^{\circ} \mathrm{C}, 2 \mathrm{~h}\right)$ and the supernatant (cell-free extract) removed. A series of ammonium sulphate precipitations were carried out on the supernatant and the precipitates forming at $30 \%$ and $40 \%$ saturation were combined and dissolved in $3 \mathrm{ml}$ buffer A. The solution was applied to a DEAE-Sepharose column connected to a BioLogic HP system equilibrated in buffer A. Proteins were eluted by applying the following salt gradient: (i) $0-0.2 \mathrm{M} \mathrm{NaCl}$ for $15 \mathrm{~min}$, (ii) $0 \cdot 2 \mathrm{M} \mathrm{NaCl}$ for $15 \mathrm{~min}$, (iii) $0 \cdot 2-0 \cdot 6 \mathrm{M} \mathrm{NaCl}$ for $180 \mathrm{~min}$, (iv) $0 \cdot 6-1 \cdot 0 \mathrm{M} \mathrm{NaCl}$ for $15 \mathrm{~min}$. CheV2-enriched fractions eluted at $0 \cdot 25-0.35 \mathrm{M} \mathrm{NaCl}$. These were pooled, concentrated to about $4 \mathrm{ml}$ and applied to a Superdex 75 gelfiltration column equilibrated in $10 \mathrm{mM}$ HEPES $\mathrm{pH} 7 \cdot 5$ containing $0 \cdot 2 \mathrm{M} \mathrm{NaCl}$ (buffer B) connected to a Pharmacia FPLC system. Proteins were eluted in $2 \mathrm{ml}$ fractions at $2 \mathrm{ml}$ $\mathrm{min}^{-1}$ in buffer B for $60 \mathrm{~min}$. Purification was monitored by SDS-PAGE and fractions containing pure CheV2 were pooled and concentrated to $1-2 \mathrm{ml}$. N-terminal sequencing by the automated Edman degradation method (carried out by Dr A. J. G. Moir in the authors' Department) was used to confirm the identity of the purified protein.

Fluorescence spectroscopy. This was performed using a Hitachi F-2500 spectrofluorimeter with an excitation wavelength of $280 \mathrm{~nm}$ and an emission wavelength of $340 \mathrm{~nm}$. The assay mixture consisted of $0 \cdot 1-0 \cdot 2 \mu \mathrm{M}$ CheV2 in $1.5 \mathrm{ml} 10 \mathrm{mM}$ HEPES pH 7.5/1 mM EDTA/1 mM magnesium chloride (buffer C). The sample cuvette was maintained at $25^{\circ} \mathrm{C}$ in the spectrofluorimeter housing. Fluorescence changes upon the addition of acetyl phosphate $(0 \cdot 5-50 \mathrm{mM}$ final concentration) were monitored until the fluorescence change stabilized. Fluorescence values were corrected for dilution.

Protein sequence and phylogenetic analyses. Multiple sequence analyses were performed using the UWGCG program PILEUP (Devereux et al., 1984). For phylogenetic analyses, sequences were aligned in CLUSTAL $\mathrm{x}$ (Thompson et al., 1997) and the output file used in PHYLIP (Felsenstein, 1993) to produce a distance matrix tree with the confidence level of the tree topology determined by bootstrapping (100 datasets). The final trees were viewed in TREEVIEW (Page, 1996).

\section{RESULTS}

\section{Mutagenesis of cheV and cheW genes in $H$. pylori}

In order to determine the importance of the predicted che $V$ genes in H. pylori chemotaxis, a set of isogenic mutants in strain 26695 was constructed. Fig. 1 shows the chromosomal arrangement of the genes analysed in this study, and the strategy used for insertional mutagenesis. PCR-amplified genes cloned into pGEM$3 \mathrm{Zf}(-)$ were insertionally inactivated at unique restriction sites using antibiotic-resistance cassettes, and transferred into H. pylori 26695 by natural transformation. The strongly polar cat cassette (Wang \& Taylor, 1990) could only be used to inactivate HP0391 (cheW) and HP0616 (cheV2) as in these cases any downstream genes are either known not to be involved in chemotaxis or are transcribed with opposite polarity. Downstream of HP0019 (cheV1) is a gene (nspC) encoding a putative carboxynorspermidine decarboxylase, which has recently been shown to be essential in H. pylori (Chalker et al., 2001). Attempts to obtain chromosomal mutations in HP0019 by transformation with plasmids containing the standard (polar) cat cassette, in either orientation, were not successful. However, transformation with pMSP15, containing a non-polar cat cassette (constructed as described in Methods) in the correct orientation resulted in successful transformation of $H$. pylori, presumably as a result of readthrough into the $n s p C$ gene. The resulting cheV1 mutant showed the same growth rate as the parent strain. This non-polar cassette was also used to inactivate HP0393 (cheV3), which appears to be part of the same operon as the che AY gene, HP0392 and cheW (HP0391; Fig. 1). In addition to these single mutants, a cheV2/cheV 3 double mutant (see below) was also constructed by transformation of strain MSP300 (26695 cheV3::cat) with pMSP18, which contains the C. coli aphAIII kanamycin-resistance gene 
(a)

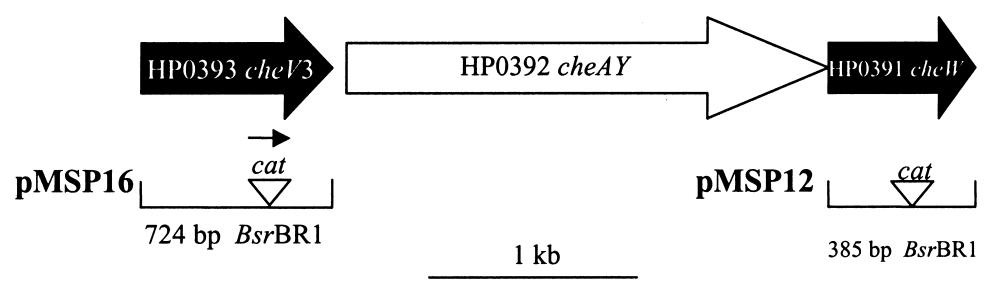

(b)

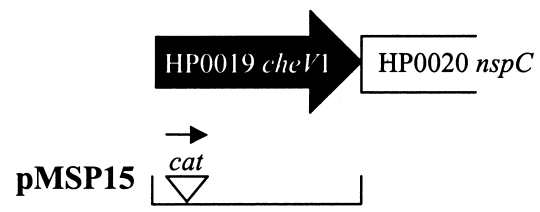

$141 \mathrm{bp} \mathrm{NcoI}$

(d)

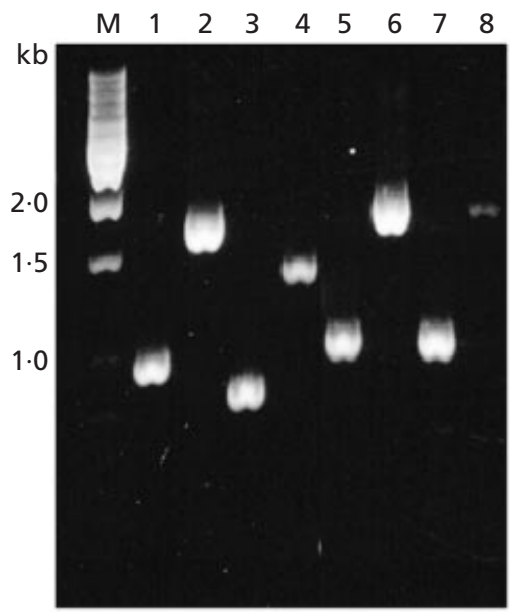

(c)

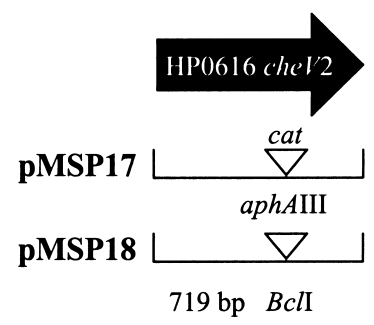

(e)

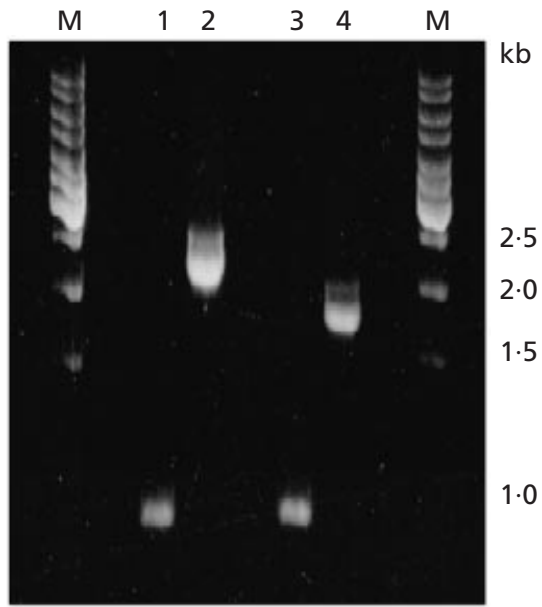

Fig. 1. Organization of cheV and cheW genes in $\mathrm{H}$. pylori 26695, mutagenesis strategy and verification of mutant strains. Mutants were constructed by cloning cat or aphAlll cassettes (inverted triangles) into unique restriction sites in the insert DNA of the relevant plasmids shown (see also Table 1). The distance (bp) from the translation start of the gene to the cassette insertion site is also shown. For cheW (a) and cheV2 (c), the standard polar cat cassette (Wang \& Taylor, 1990) was used for inactivation, while for cheV1 (b) and cheV3 (a), a cassette lacking the $3^{\prime}$ transcriptional termination signals was inserted with the same polarity as the target gene to allow readthrough into the downstream genes (indicated by the arrow above the cassette insertion site). (d) $0.8 \%$ agarose gel of PCR products obtained with the parental strain 26695 genomic DNA as template (lanes 1, 3, 5 and 7) or with DNA from cheV1 (lane 2), cheW (lane 4), cheV3 (lane 6) or cheV2 (lane 8) mutants, using primers cheV1-F and cheV1-R (lanes 1 and 2), cheW-F and cheW-R (lanes 3 and 4), cheV3-F and cheV3-R (lanes 5 and 6) or cheV2-F and cheV2-R (lanes 7 and 8). Lane M contains size markers (sizes indicated). (e) $0.6 \%$ agarose gel of PCR products obtained with the parental 26695 genomic DNA as template (lanes 1 and 3) or with DNA from the cheV2::aph/cheV3::cat strain MSP400 (lanes 2 and 4) using primers cheV2-F and cheV2-R (lanes 1 and 2) or cheV3-F and cheV3-R (lanes 3 and 4). In (d) and (e) the increase in size of the single PCR products obtained when mutant DNA is used as the template indicates the correct insertion of the cat or aphlll cassettes.

(Wang \& Taylor, 1990) inserted at the unique BclI restriction site in HP0616. For each mutant, verification that correct chromosomal insertion of the cassettes had occurred by a double homologous recombination event was obtained by PCR analysis of parental and mutant genomic DNA (Fig. 1). In each case, a single PCR product of the predicted molecular size was obtained.

Electron microscopy of negatively stained cells showed that each of the mutants constructed had a normal 

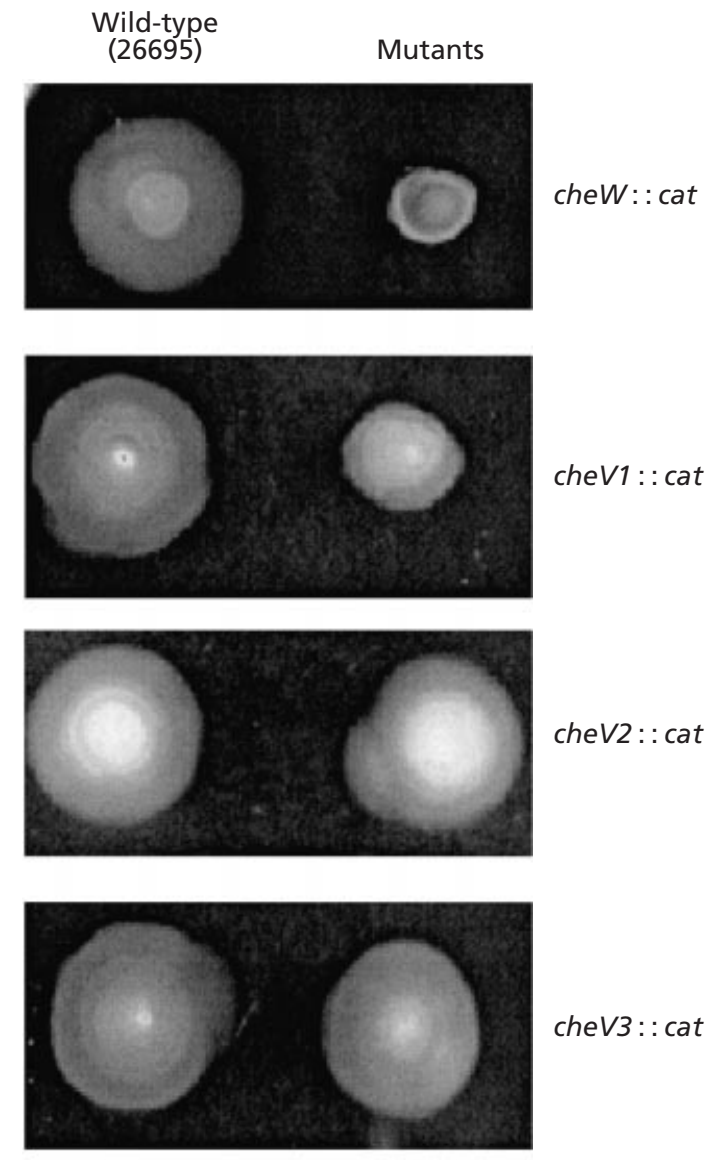

cheV3 : : cat

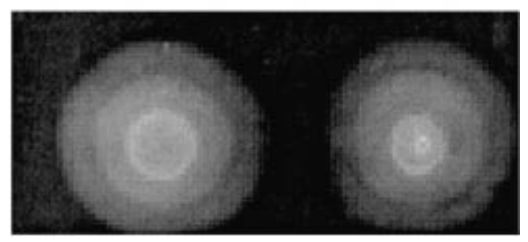

cheV2::aph/ cheV3: : cat

Fig. 2. Chemotaxis of $H$. pylori wild-type 26695 (left of each panel) and mutant strains (right of each panel) indicated by swarming ability on soft agar. Overnight $\mathrm{BHI}$ broth cultures $(10 \mu \mathrm{l})$ of $H$. pylori strains were spotted onto the surface of dried $\mathrm{BHI}$ plates containing $0.32 \%(\mathrm{w} / \mathrm{v})$ agar. The plates were incubated microaerobically for $5 \mathrm{~d}$ at $37^{\circ} \mathrm{C}$ and then photographed. The establishment of a nutrient gradient during growth results in chemotactic swarming of the cells outward from the inoculum site, forming characteristic concentric rings. Cells which are deficient in chemotaxis do not swarm and remain close to the site of inoculation.

pattern and number of flagella, indicating that there was no perturbation of flagellar biosynthesis or assembly. Moreover, the motility of strain 26695 and the mutants examined under phase-contrast microscopy was similar in terms of the percentage of motile cells, and the overall level of swimming activity (data not shown).

The chemotactic ability of the $H$. pylori mutants was determined by swarm-plate assays on semi-solid BHI medium (Fig. 2). The formation of a visible swarm surrounding the site of inoculation results from the production of a nutrient gradient during growth, to which the cells respond chemotactically by moving outwards, forming characteristic concentric rings. Nonchemotactic cells will remain near the site of inoculation. The parental strain 26695 consistently formed large swarms after 3-4 d growth (Fig. 2). However swarming was abolished in the HP0391 (cheW) mutant (Fig. 2); the cells only grew at the site of inoculation and did not move outwards to any significant extent. This gene is therefore essential for H. pylori chemotaxis. Swarming was also severely reduced in the HP0019 (cheV1) mutant in comparison to the parental strain (Fig. 2), but was not completely abolished. In contrast, mutations in HP0616 (cheV2) or HP0393 (cheV3) did not result in any decrease in swarming ability compared to the isogenic wild-type parent (Fig. 2). A cheV2/cheV3 double mutant was constructed to test whether these genes can replace each other's function. However, this strain also clearly exhibited normal swarming behaviour (Fig. 2). The restriction sites used for insertion of the cat cassette into cheV 2 and cheV 3 are located towards the $3^{\prime}$ end of the genes, which might result in the synthesis of partially functional truncated and/or fusion proteins containing the CheW domains. In order to rule out this possibility, the expression in E. coli of CheV from the IPTGinducible ptac promoter in pMSP10 and pMSP11 was compared to that of the same plasmids containing the cat-inactivated genes. Whereas IPTG-inducible proteins of the predicted size for $\mathrm{CheV}(33 \mathrm{kDa})$ were expressed in the case of the wild-type genes, these were clearly absent in the mutant constructs and no truncated or fusion proteins were observed (data not shown). Overall, the mutant phenotypes suggest that cheV1 plays an important role in chemotaxis and that its function cannot be substituted for by either cheV2 or cheV3.

\section{Effect of expression of $\boldsymbol{H}$. pylori chemotaxis genes in E. coli wild-type and chemotaxis mutants}

To further characterize the function of the che $V$ and cheW genes in H. pylori, attempts were made to complement E. coli chemotaxis mutants by expression of the heterologous genes and determine the effect of expression on chemotaxis in the wild-type. The following E. coli strains were used: the parental strain RP437, which showed normal chemotaxis and swarming

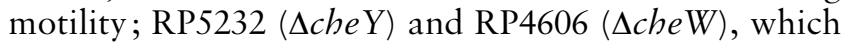
both had a smooth-swimming phenotype. These mutants were confirmed to be non-chemotactic by swarm plate assays. The H. pylori cheW, cheV1, cheV2 and $c h e V 3$ genes were amplified by PCR and cloned into the pTM30 expression vector (Morrison \& Parkinson, 1994), which allows controlled expression from a ptac promoter using IPTG concentrations in the range $0-1000 \mu \mathrm{M}$. The constructs (see Table 1) were transformed into RP437. IPTG-dependent expression of the CheW, CheV2 and CheV3 proteins was confirmed by SDS-PAGE analysis, but CheV1 expression from pMSP8 was weak and IPTG independent (data not shown), and so this plasmid was not used further. RP5232 ( $\Delta$ che Y) and RP4606 ( $\Delta$ cheW) transformed with pMSP10 or pMSP11, bearing the H. pylori cheV 3 and cheV2 genes 


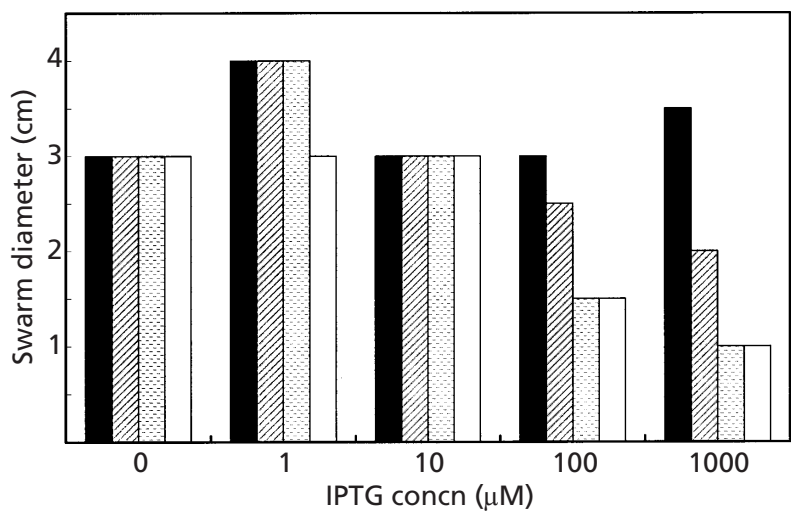

Fig. 3. Effect of heterologous expression of $H$. pylori chemotaxis genes on E. coli chemotaxis. RP437 was transformed with pTM30 (black bars) or with pTM30-containing $\mathrm{H}$. pylori cheV3 (pMSP10; diagonal striped bars), cheV2 (pMSP11; dashed bars) or cheW (pMSP9; clear bars). Overnight LB cultures of the transformants $(10 \mu \mathrm{l})$ were spotted onto the surface of LB plates containing $0.3 \%(\mathrm{w} / \mathrm{v})$ agar and the concentrations of IPTG indicated, and incubated at $30^{\circ} \mathrm{C}$ for $8 \mathrm{~h}$, after which time the diameters of the swarms were measured. The experiment was repeated several times with similar results; the results of a representative experiment are shown.

respectively, did not show restoration of swarming at any level of IPTG induction from 0 to $1 \mathrm{mM}$. Similarly, expression of CheW from pMSP9 in RP4606 ( $\Delta$ cheW) also did not complement the swarming phenotype of this strain at any IPTG concentration up to $1 \mathrm{mM}$. However, expression of cheW, cheV2 and cheV 3 in the parental strain RP437 had clear IPTG concentration dependent inhibitory effects on chemotaxis (Fig. 3). The most dramatic effects were observed with cheW and cheV2, where swarming was severely reduced at high levels of induction (Fig. 3), but expression of cheV 3 also resulted in significant inhibition of swarming in E. coli (Fig. 3).

\section{Phylogenetic and sequence analysis of CheV paralogues: conservation of active-site residues in the CheY-like $\mathrm{N}$-terminal domain}

$\mathrm{CheV}$ proteins are present in only some Gram-positive and Gram-negative bacteria but not in Archaea. Fig. 4(a) shows the phylogenetic relationships between the three CheV paralogues in H. pylori 26695 and all of the $\mathrm{CheV}$ protein sequences present in the databases to date. Bacillus halodurans, Vibrio cholerae and H. pylori all contain more than one cheV gene and in these bacteria the encoded paralogues are generally quite divergent from each other. This is particularly apparent in the case of V. cholerae. In H. pylori, the three paralogues share less than $30 \%$ sequence identity and appear to have diverged differently. HP0393 is most closely related to the single $\mathrm{CheV}$ protein in C. jejuni, which is also part of a cheV-cheAY-cheW operon. HP0019 and HP0616 are more similar to each other than either is to HP0393 although they have also diverged significantly from each other during evolution.
Of particular interest for the function of $\mathrm{CheV}$ is the presence of a C-terminal CheY-like domain in this protein. Fig. 4(b) shows a sequence alignment of the Cterminal domains of the $H$. pylori paralogues with the $E$. coli and $H$. pylori CheY proteins. The highly conserved residues that form the active site of the E. coli CheY are present or are replaced with conservative substitutions in all three H. pylori $\mathrm{CheV}$ proteins. These include D57, the site of phosphorylation (Sanders et al., 1989a), D12 and D13 (D12 is replaced by a glutamate in CheV2), which are involved in the co-ordination of $\mathrm{a} \mathrm{Mg}^{2+}$ ion which is essential for phosphorylation and dephosphorylation to occur (Volz, 1993), and K109, involved in the phosphorylation-induced conformational change (Volz, 1993). The position corresponding to T87 in $H$. pylori and E. coli CheY molecules is occupied by a hydroxy amino acid (threonine or serine) in virtually all known response regulators (Volz, 1993). In the CheY domains of the three CheV's, the corresponding T87 residue is replaced by serine. It is clear, therefore, that the CheY domains of each of the CheV proteins in $H$. pylori could potentially accept phosphoryl groups.

\section{Evidence for phosphorylation of CheV2 by acetyl phosphate}

In order to gain evidence for phosphorylation of the CheY domains of the CheV proteins, E. coli cultures harbouring pMSP8, pMSP10 or pMSP11 were induced with $1 \mathrm{mM}$ IPTG, harvested, and attempts made to purify to homogeneity the overexpressed CheV proteins. Insufficient amounts of $\mathrm{CheV} 1$ and $\mathrm{CheV} 3$ were expressed from pMSP8 and pMSP10, respectively, for successful purification, but CheV2 was optimally expressed to about $20 \%$ of the soluble protein (Fig. 5a) and was successfully purified by a combination of ionexchange and gel-filtration chromatography after an initial ammonium sulphate fractionation of crude cellfree extracts (Fig. 5b). The $\mathrm{N}$-terminal sequence of the purified protein was determined to be MLQDPVRDIDKTT. Residues 6-13 are identical to residues 2-9 of the deduced $\mathrm{CheV} 2$ protein (residues 1-5 arise from the in-frame cloning into the pTM30 vector; see Methods). In view of the presence of the appropriate conserved residues in the CheY-like domains of each of the proteins, CheV2 was considered to be a suitable representative paralogue for biochemical studies.

The phosphorylation of response-regulators such as CheY and PhoB by small-molecule phosphodonors has been successfully studied by fluorescence spectroscopy, exploiting the tryptophan residue adjacent to the phospho-accepting active-site aspartate residue in these proteins (Lukat et al., 1992; McCleary, 1996; Fig. 4b). The corresponding residue in CheV2 is a leucine (Fig. 4b); there are two Trp residues in the protein, but both are in the N-terminal CheW domain. However, F255 may be a suitable reporter of phosphorylation; the corresponding region in the E. coli CheY undergoes a large change of environment upon phosphorylation (Stock \& Surette, 1996). The fluorescence emission 
(a)

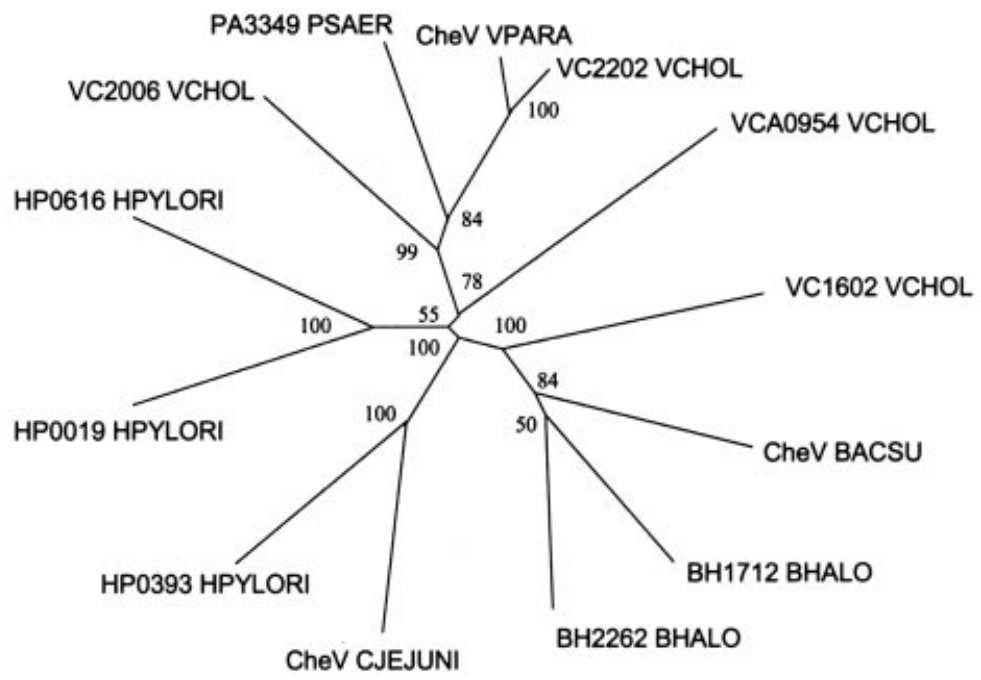

0.1

(b)
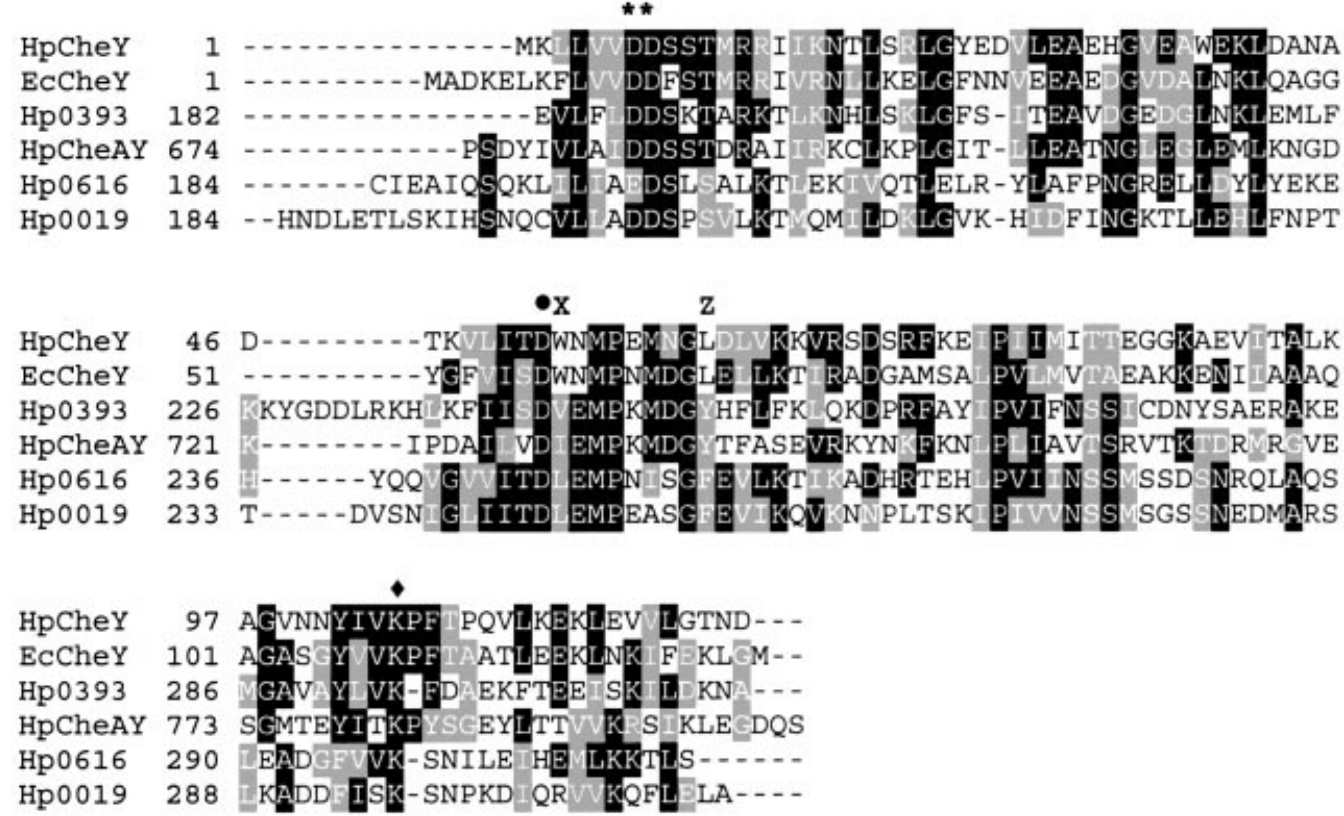

Fig. 4. Phylogenetic relationships of $\mathrm{CheV}$ proteins and sequence conservation in the $\mathrm{C}$-terminal CheY-like domains of the $H$. pylori proteins. In (a) a CLUSTAL x multiple sequence alignment of all of the CheV homologues was used as the input to PHYLIP to generate a bootstrapped distance matrix tree (nodal bootstrap values indicated). The bar represents 1 inferred substitution per 10 residues. PSAER, Psuedomonas aeruginosa; VPARA, Vibrio parahaemolyticus; VCHOL, Vibrio cholerae; BACSU, Bacillus subtilis; BHALO, Bacillus halodurans; CJEJUNI, Campylobacter jejuni; HPYLORI, Helicobacter pylori. (b) A PILEUP alignment of the CheY domains of the three H. pylori CheV paralogues (HP0019, HP0393 and HP0616), the CheY domain of the CheAY protein (HpCheAY), the $H$. pylori CheY protein itself (HpCheY) and the E. coli CheY $(\mathrm{EcCheY})$ is shown. Identical residues are boxed in black and similar residues are boxed in grey. Key $E$. coli CheY active-site residues D12, D13 (asterisks), D57 (the site of phosphorylation; filled circle) and K109 (filled diamond) are conserved in all of the $H$. pylori CheY domains. $X$ indicates the position of the tryptophan residue in the $E$. coli CheY which reports conformational changes upon phosphorylation by fluorescence quenching (Lukat et al., 1992). Although this residue is conserved in $\mathrm{H}$. pylori CheY, it is replaced by aliphatic hydrophobic amino acids in all of the CheV proteins. A nearby phenylalanine residue in HP0616 (Z) may be responsible for the fluorescence changes observed in the purified protein (see Fig. 6). 


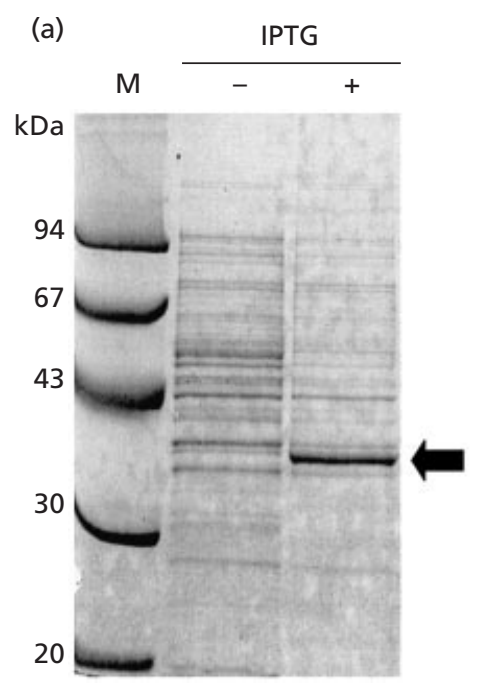

(b)

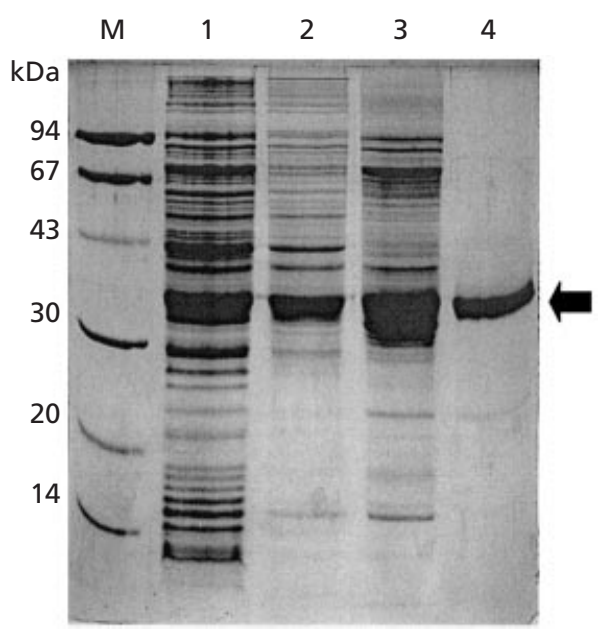

Fig. 5. Overexpression and purification of the $H$. pylori CheV2 protein (HP0616). In (a), two $E$. coli DH5 $\alpha$ (pMSP11) cultures were grown aerobically in LB to an $\mathrm{OD}_{600}$ of 0.5 . IPTG $(1 \mathrm{mM})$ was then added to one culture and incubation continued for $5 \mathrm{~h}$. Cells were then harvested and subjected to SDS-PAGE as described in Methods. The Coomassie-blue-stained gel shows the IPTG-dependent expression of the $33 \mathrm{kDa}$ CheV2 protein (arrow). Molecular mass markers (sizes indicated) were run in lane M. (b) Purification of CheV2. Lane M contains molecular mass markers. Lane 1, cell-free extract from DH5 $\alpha$ (pMSP11) after $5 \mathrm{~h}$ induction with IPTG. Lane 2, 30-40\% saturation ammonium sulphate precipitate. Lane 3, fractions from DEAE-Sepharose chromatography eluting at $0.25-0.35 \mathrm{M} \mathrm{NaCl}$. Lane 4 , fractions from Superdex-75 gel filtration chromatography. The arrow indicates the $33 \mathrm{kDa}$ CheV2 protein.

spectrum of CheV2 is shown in Fig. 6(a). The fluorescence properties of CheV2 were characterized by excitation and emission maxima of $280 \mathrm{~nm}$ and $340 \mathrm{~nm}$, respectively. The excitation maximum reflects the predominance of Tyr and Phe over Trp residues in the protein. Addition of acetyl phosphate to the protein in the presence of $\mathrm{Mg}^{2+}$ ions caused a significant quench in fluorescence, with no change in the emission maximum (Fig. 6a). The dependence of the fluorescence change on acetyl phosphate concentration was hyperbolic (Fig. 6b) and the maximum fluorescence quench at $50 \mathrm{mM}$ acetyl phosphate was about $5 \%$. CheV was considered to be an acetyl-phosphatase with phospho-CheV as an intermediate, in an analogous manner to E. coli CheY (Lukat et al., 1992) :

$\mathrm{CheV}+\mathrm{Ac} \sim \mathrm{P} \stackrel{K_{\mathrm{s}}}{\leftrightarrow}[\mathrm{CheV}-\mathrm{Ac} \sim \mathrm{P}] \stackrel{k_{2}}{\rightarrow} \mathrm{CheV} \sim \mathrm{P} \stackrel{k_{3}}{\rightarrow} \mathrm{CheV}+\mathrm{P}_{\mathrm{i}}$

Thus the $K_{\mathrm{m}}$ of CheV2 for acetyl phosphate is equal to $K_{\mathrm{s}} k_{3} / k_{2}$ in this scheme and can be determined from the fluorescence change assuming that the observed quenching is a direct effect of the reduced quantum yield of phospho-CheV2 relative to CheV2 (Lukat et al., 1992). The reciprocal of the slope of the line in Fig. 6(c) gave a $K_{\mathrm{m}}$ value of $21 \mathrm{mM}$.

\section{DISCUSSION}

H. pylori contains a complex chemotaxis system, with a unique combination of proteins including the fused CheAY histidine-kinase/response-regulator and three CheV paralogues (Kelly, 1998; Foynes et al., 2000). In this study we have focussed on determining the essentiality and possible function of the latter proteins.
The presence of the $\mathrm{N}$-terminal CheW domain in all CheV proteins may suggest a function in the early stages of signal transduction, from the receptors to CheA. Indeed, Rosario et al. (1994) showed that partial functional redundancy exists between the $B$. subtilis $\mathrm{CheV}$ and $\mathrm{CheW}$ proteins, as expression of the $\mathrm{N}$ terminal domain of $\mathrm{CheV}$ was sufficient to rescue the chemotactic defect of a cheW mutant of this organism. In H. pylori, we have shown that cheW is an essential chemotaxis gene, most likely because CheW links the receptors to activation of CheA, as in E. coli, but the role of CheW cannot apparently be replaced by any of the three $\mathrm{CheV}$ proteins in a cheW mutant background. Overexpression of $H$. pylori CheW in E. coli inhibited swarming and induced a smooth-swimming phenotype in the wild-type strain RP437. This is the same effect seen upon overexpression of the homologous E. coli CheW (Sanders et al., 1989b). The production of a molar excess of CheW may result in inhibition of the interaction required to catalyse CheA autophosphorylation, possibly by excess $\mathrm{CheW}$ displacing the CheWCheA complex from the receptor (Hamblin et al., 1997). The result would be the formation of an inactive complex that cannot initiate the flow of phosphate to CheY. This would reduce the levels of phospho-CheY, resulting in tumble suppression. Hamblin et al. (1997) successfully complemented an E. coli $\Delta$ cheW mutant (RP4606) with cheW from Rhodobacter sphaeroides, which is a member of the $\alpha$-subgroup of Proteobacteria. In contrast, the $H$. pylori cheW gene was apparently unable to complement RP4606. Lack of complementation may be due to insufficient functional similarity, or to an unsuitable level of CheW overexpression 

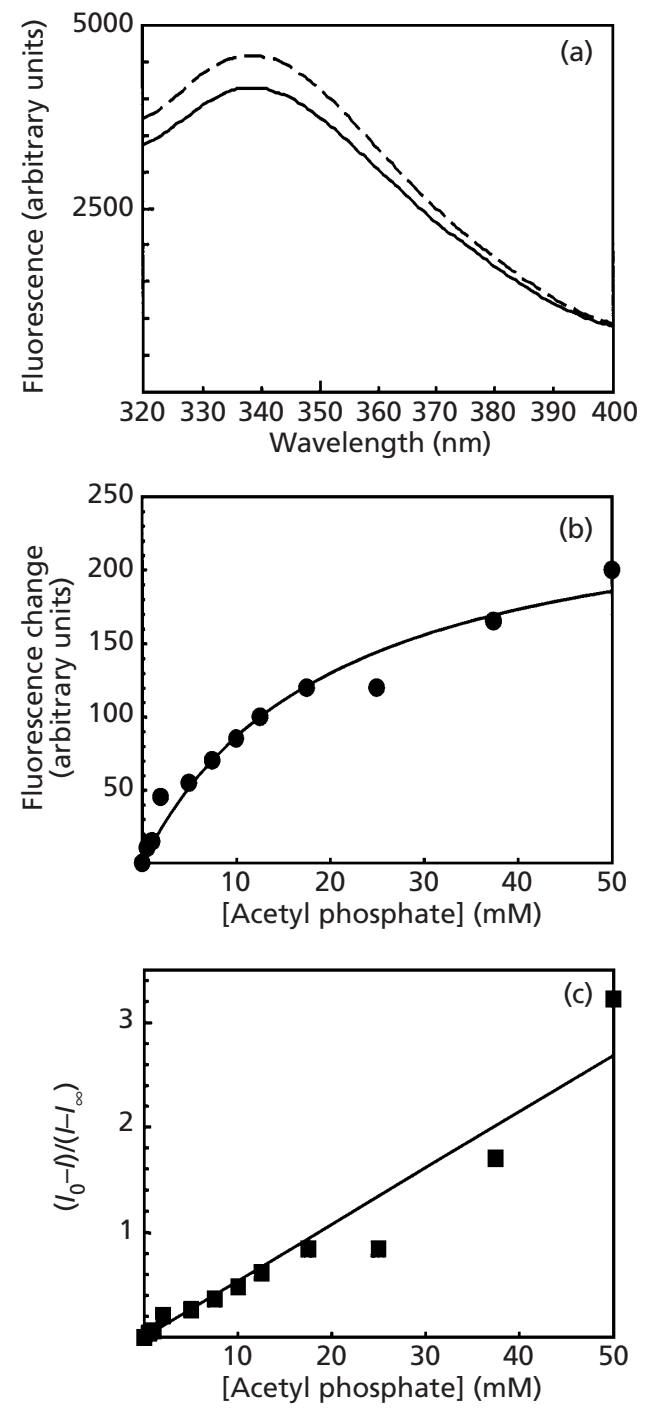

Fig. 6. Fluorescence changes in CheV2 upon addition of acetyl phosphate. (a) Fluorescence emission spectrum of $0.2 \mu \mathrm{M}$ $\mathrm{CheV2}$, at an excitation wavelength of $280 \mathrm{~nm}$. Dashed line, protein fluorescence in the absence of acetyl phosphate; solid line, fluorescence in the presence of $50 \mathrm{mM}$ acetyl phosphate. (b) Titration of fluorescence change with acetyl phosphate. The CheV2 concentration was $0.1 \mu \mathrm{M}$ in buffer C. (c) Determination of $K_{\mathrm{m}}$ for acetyl phosphate. The ratio of the acetyl phosphateinduced fluorescence quench $\left(I_{0}-l\right.$, where $I$ is the fluorescence intensity at a given acetyl phosphate concentration, and $I_{0}$ is the fluorescence intensity of the $\mathrm{CheV}-\mathrm{Mg}^{2+}$ complex in the absence of acetyl phosphate) and the difference between the fluorescence intensity at saturating acetyl phosphate and I (i.e. $I-I_{\infty}$ ) is equal to [acetyl phosphate] $k_{2} / k_{3} K_{\mathrm{s}}$ by analogy with the reaction scheme for CheY proposed by Lukat et al. (1992) and described in the text for $\mathrm{CheV}$. Thus the reciprocal of the slope of the line relating this ratio to the phosphodonor concentration gave a $K_{\mathrm{m}}$ value of $21 \mathrm{mM}$ acetyl phosphate.

(Sanders et al., 1989b). Protein stoichiometry is important in chemotaxis, and although the pTM30 vector gives graded expression with IPTG, the lowest concentration of IPTG used $(1 \mu \mathrm{M})$ may still have induced a molar excess of this protein over the other chemotaxis proteins in E. coli.
The results from insertional inactivation of each of the three cheV genes in $H$. pylori revealed that CheV1 is critical for chemotaxis, as a cheV1 null mutant was severely affected in its ability to swarm on soft agar, although swarming was not completely abolished. The expression of the two other cheV genes may be responsible for this residual level of swarming. However, the single cheV2 and cheV3 mutants were fully chemotactic and formed swarms on semi-solid agar indistinguishable from those of the parental strain. These genes cannot simply substitute for each other's function, as a cheV $2 / \mathrm{cheV} 3$ double mutant strain was also fully chemotactic. In the double mutant it seems likely that CheV1 can subsitute for the roles of both $\mathrm{CheV} 2$ and $\mathrm{CheV} 3$. The results suggest a dominant and specialized function for CheV1 in H. pylori chemotaxis. The expression of H. pylori cheV 2 or cheV 3 in the E. coli $\Delta c h e W$ or $\Delta c h e Y$ mutants did not restore a swarming phenotype. However, as with cheW, when expressed in the isogenic $E$. coli wild-type strain these genes caused a clear inhibition of swarming. Therefore, $\mathrm{CheV} 2$ and CheV3 can interact with the E. coli chemotaxis system but as they consist of both a CheW and a CheY domain, the mechanism of interaction may be complex. The CheW domains could bind to the chemoreceptors and inhibit chemotaxis in the same manner as that described for the H. pylori CheW. However, the CheY domains could also compete with the native E. coli CheY for phosphate from CheA. The overall effect of this would be to sequester phosphoryl groups from the native $E$. coli CheY, thus causing a build-up of unphosphorylated E. coli CheY which would be unable to bind to the motor switch. The net effect would be that the cells would tumble rarely, giving a smooth-swimming phenotype and an inability to swarm. The ability of CheV2 and $\mathrm{CheV} 3$ to perturb chemotaxis in E. coli demonstrates that these proteins do have a role in chemotaxis, despite the fact that normal swarming behaviour was still observed for $\mathrm{H}$. pylori cheV2 and cheV 3 mutants. However, in this study we have not examined the swimming patterns of the $H$. pylori mutants in detail, and it would be informative to study their behaviour by video tracking, to reveal any motility defects not apparent in simple swarm assays.

Thus, the most likely roles for the $\mathrm{CheV}$ proteins in $H$. pylori could involve the ability of their CheY domains to be phosphorylated. All of the proteins contain the necessary active-site residues, and we have shown using fluorescence spectroscopy that $\mathrm{CheV2}$, as a representative of the CheV family, is able to bind the smallmolecule phosphodonor acetyl phosphate in a similar manner to that which is characteristic of CheY and other response-regulators (Lukat et al., 1992; McCleary, 1996). This is the first evidence for phosphorylation of any $\mathrm{CheV}$ protein. The low affinity observed indicates that acetyl phosphate itself is not physiologically important as a phosphodonor to $\mathrm{CheV}$ in vivo; the current view of E. coli CheY from stopped-flow kinetic studies of acetyl phosphate binding is that CheA provides a high local concentration of phosphate groups (Da Re et al., 
1999). Phosphorylation of $\mathrm{CheV}$ proteins may function in controlling the flow of phosphate to CheY itself, by the $\mathrm{CheV}$ proteins acting as 'phosphate sinks'. The Nterminal CheW domain of $\mathrm{CheV}$ may interact with a site on the cytoplasmic signalling domain of the receptors and catalyse phosphoryl transfer from CheA onto the Cterminal CheY domain of the protein. The receptorbinding site may be the same as used by the CheW protein, thus inhibiting the flow of phosphate to the CheY protein itself by a process of competitive binding. The need for a phosphate sink may be related to the absence of a cheZ homologue in H. pylori, as is the case with other non-enteric bacteria. For example, there are no cheZ homologues in $R b$. sphaeroides and Sinorhizobium meliloti but these organisms do possess several copies of cheY (Armitage \& Schmitt, 1997; Sourjik \& Schmitt, 1996). A phosphate sink function to control phospho-CheY levels has been suggested for these additional CheY proteins (Armitage \& Schmitt, 1997) and for the CheY domain of CheAY in H. pylori (Foynes et al., 2000). Our results suggest that the CheY domains of the $\mathrm{CheV}$ proteins also have an important role as phospho-acceptors.

The purification of all of the H. pylori chemotaxis proteins, and the reconstitution of the signal-transduction cascade in vitro to demonstrate how intramolecular and intermolecular phosphotransfer from CheAY to the other CheY domains occurs, will be needed to fully explain the role and mechanism of the individual $\mathrm{CheV}$ proteins, and to further our understanding of this novel chemotaxis system.

\section{ACKNOWLEDGEMENTS}

This work was supported by a studentship from the UK Medical Research Council to M.S.P. and a Biotechnology and Biological Sciences Research Council grant to D.J.K. for M. G. We thank Dr Gavin Thomas for help with phylogenetic analysis, Professor Judith Armitage and Dr Paul Hamblin for strains, and Douglas Brown and Matthew Wand for their help with the fluorescence studies.

\section{REFERENCES}

Akopyants, N. S., Eaton, K. A. \& Berg, D. E. (1995). Adaptive mutation and co-colonisation during infection of gnotobiotic piglets. Infect Immun 63, 116-121.

Alm, R. A., Ling, L.-S. L., Moir, D. T. \& 20 other authors (1999). Genomic sequencing comparison of two unrelated isolates of the human gastric pathogen Helicobacter pylori. Nature 397, 176180.

Armitage, J. P. \& Schmitt, R. (1997). Bacterial chemotaxis: Rhodobacter sphaeroides and Sinorhizobium meliloti-variations on a theme? Microbiology 143, 3671-3682.

Beier, D., Spohn, G., Rappuoli, R. \& Scarlato, V. (1997). Identification and characterisation of an operon of Helicobacter pylori that is involved in motility and stress adaptation. J Bacteriol $\mathbf{1 7 9}$, 4676-4683.

Blair, D. F. (1995). How bacteria sense and swim. Annu Rev Microbiol 49, 489-522.
Bourret, R. B., Borkovich, K. A. \& Simon, M. I. (1991). Signal transduction involving phosphorylation in prokaryotes. Annu Rev Biochem 60, 401-441.

Chalker, A. F., Mineheart, H. W., Hughes, N. J. \& 8 other authors (2001). Systematic identification of selective essential genes in Helicobacter pylori by genome prioritization and allelic replacement mutagenesis. J Bacteriol 183, 1259-1268.

Da Re, S. S., Deville-Bonne, D., Tolstyk, T., Veron, M. \& Stock, J. B. (1999). Kinetics of CheY phosphorylation by small molecule phosphodonors. FEBS Lett 457, 323-326.

Devereux, J., Haeberli, P. \& Smithies, O. (1984). A comprehensive set of sequence analysis programs for the VAX. Nucleic Acids Res 12, 387-395.

Doig, P., de Jonge, B. L., Alm, R. A. \& 10 other authors (1999). Helicobacter pylori physiology predicted from genomic comparison of two strains. Microbiol Mol Biol Rev 63, 675-707.

Eaton, K. A., Morgan, D. R. \& Krakowa, S. (1992). Motility as a factor in the colonisation of gnotobiotic piglets by Helicobacter pylori. J Med Microbiol 37, 123-127.

Eaton, K. A., Suerbaum, S., Josenhans, C. \& Krakowa, S. (1996). Colonisation of gnotobiotic piglets by Helicobacter pylori deficient in two flagellin genes. Infect Immun 64, 2445-2448.

Felsenstein, J. (1993). PHYLIP: Phylogeny Inference Package. Version 3.5. Seattle, WA: University of Washington.

Ferrero, R. L., Cussac, V., Courcoux, P. \& Labigne-Roussel (1992). Construction of isogenic urease-negative mutants of Helicobacter pylori by allelic exchange. J Bacteriol 174, 4212-4217.

Forman, D., Newell, D. G., Fullerton, F., Yarnell, J. W. G., Stacey, A. R., Wald, N. \& Sitas, F. (1991). Association between infection with Helicobacter pylori and risk of gastric cancer : evidence from a perspective investigation. $\mathrm{Br}$ Med J 302, 1302-1305.

Foynes, S., Dorrell, N., Ward, S. J., Stabler, R. A., McColm, A. A., Rycroft, A. N. \& Wren, B. W. (2000). Helicobacter pylori possesses two CheY response regulators and a histidine kinase sensor, CheA, which are essential for chemotaxis and colonization of the gastric mucosa. Infect Immun 68, 2016-2023.

Frederick, K. L. \& Helmann, J. D. (1994). Dual chemotaxis signalling pathways in Bacillus subtilis: a $\sigma^{\mathrm{D}}$-dependent gene encodes a novel protein with both $\mathrm{CheW}$ and $\mathrm{CheY}$ homologous domains. J Bacteriol 176, 2727-2735.

Hamblin, P. A., Bourne, N. A. \& Armitage, J. P. (1997). Characterisation of the chemotaxis protein $\mathrm{CheW}$ from Rhodobacter sphaeroides and its effect on the behaviour of Escherichia coli. Mol Microbiol 24, 41-51.

Jackson, C. J., Kelly, D. J. \& Clayton, C. L. (1995). The cloning and characterisation of chemotaxis genes in Helicobacter pylori. Gut 37(S1), A18.

Kelly, D. J. (1998). The physiology and metabolism of the human gastric pathogen Helicobacter pylori. Adv Microb Physiol 40, 137-189.

Lukat, G. S., McCleary, W. R., Stock, A. M. \& Stock, J. B. (1992). Phosphorylation of bacterial response regulator proteins by lowmolecular weight phosphodonors. Proc Natl Acad Sci US A 89, 718-722.

McCleary, W. R. (1996). The activation of PhoB by acetylphosphate. Mol Microbiol 20, 1155-1163.

McCleary, W. R. \& Stock, J. B. (1994). Acetyl-phosphate and the activation of two-component response-regulators. J Biol Chem 269, 31567-31572.

Marmur, J. (1961). A procedure for the isolation of deoxyribonucleic acid from microorganisms. J Mol Biol 3, 208-218. 
Marshall, B. J., Armstrong, J. A., McGechie, D. B. \& Glancy, R. J. (1985). Attempt to fulfil Koch's postulates for pyloric campylobacter. Med J Aust 142, 436-439.

Morrison, T. B. \& Parkinson, J. S. (1994). Liberation of an interaction domain from the phosphotransfer region of CheA, a signaling kinase of Escherichia coli. Proc Natl Acad Sci US A 91, 5485-5489.

Moss, S. \& Calam, J. (1992). Helicobacter pylori and peptic ulcers: the present position. Gut 33, 289-292.

Page, R. D. M. (1996). Treeview: an application to display phylogenetic trees on personal computers. Comput Appl Biosci 12, 357-358.

Rosario, M. M. L., Frederick, K. L., Ordal, G. W. \& Helmann, J. D. (1994). Chemotaxis in Bacillus subtilis requires either of two functionally redundant $\mathrm{CheW}$ homologues. J Bacteriol 176, 2736-2739.

Sanders, D. A., Gillece-Castro, B. L., Stock, A. M., Burlingame, A. L. \& Koshland, D. E., Jr (1989a). Identification of the site of phosphorylation of the chemotaxis protein response regulator, CheY. J Biol Chem 264, 21770-21778.

Sanders, D. A., Mendez, B. \& Koshland, D. E., Jr (1989b). Role of the $\mathrm{CheW}$ protein in bacterial chemotaxis: overexpression is equivalent to absence. J Bacteriol 171, 6271-6278.

Sourjik, V. \& Schmitt, R. (1996). Different roles of CheY1 and CheY 2 in the chemotaxis of Rhizobium meliloti. Mol Microbiol 22, 427-436.
Stock, J. B. \& Surette, M. G. (1996). Chemotaxis. In Escherichia coli and Salmonella typhimurium, pp. 1103-1129. Edited by F. C. Neidhardt and others. Washington, DC: American Society for Microbiology.

Studier, F. W. \& Moffat, B. A. (1986). Use of bacteriophage T7 RNA polymerase to direct selective high-level expression of cloned genes. J Mol Biol 189, 113-130.

Thompson, J. D., Gibson, T. J., Plewiniak, F., Jeanmougin, F. \& Higgins, D. A. (1997). The CLuSTAL_X Windows interface: flexible strategies for multiple sequence alignment aided by quality analysis tools. Nucleic Acids Res 25, 4876-4882.

Tomb, J.-F., White, O., Kerlavage, A. R. \& 39 other authors (1997). The complete genome sequence of the gastric pathogen Helicobacter pylori. Nature 388, 539-547.

Volz, K. (1993). Structural conservation in the CheY superfamily. Biochemistry 32, 11741-11753.

Wang, Y. \& Taylor, D. E. (1990). Chloramphenicol resistance in Campylobacter coli-nucleotide-sequence, expression, and cloning vector construction. Gene 94, 23-28.

Wyatt, J. I., Rathbone, B. J., Dixon, M. F. \& Heatley, R. V. (1987). Campylobacter pyloridis and acid-induced metaplasia in the pathogenesis of duodenitis. J Clin Pathol 40, 841-848.

Received 2 March 2001; revised 4 May 2001; accepted 10 May 2001. 